\title{
Effect of milk phospholipids on the growth and cryotolerance of lactic acid bacteria cultured and stored in acid whey-based media
}

\author{
Lin Zhang, ๑ Israel García-Cano, ๑ and Rafael Jiménez-Flores* $\odot$
}

\section{Graphical Abstract}

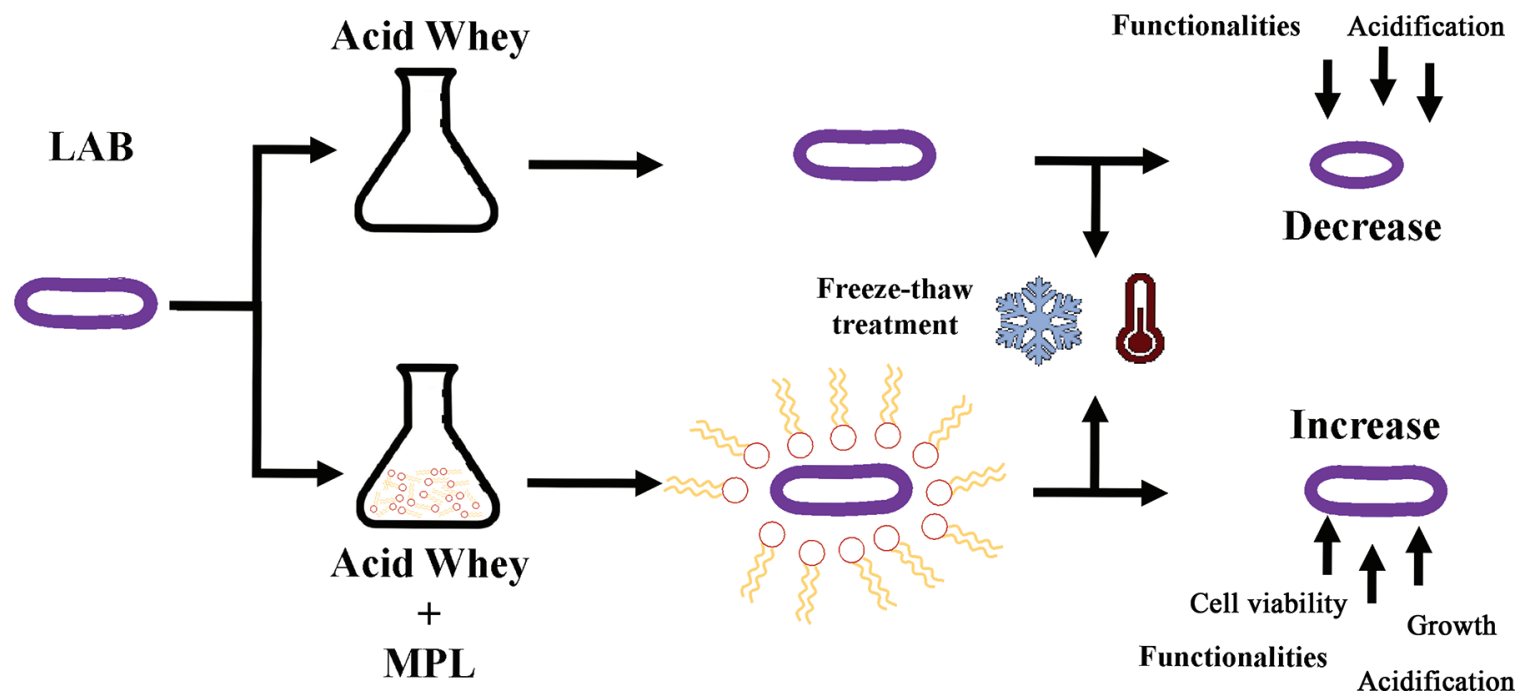

\section{Summary}

Supplementation of a lactic acid bacteria (LAB) culture medium with milk phospholipids (MPL) at low concentration $(0.5 \%)$ results in a significant increase in viability and metabolic activity after freeze-thawing cycles. We speculate that the nature of the milk phospholipids maintains the integrity of the bacterial membranes and thus their metabolic function. This work demonstrates a feasible and economic way to enhance the cryotolerance of lactic acid bacteria, pointing toward possible means to develop functional foods and valorize acid whey.

\section{Highlights}

- Acidification activity is retained by adding milk phospholipids to acid whey-based medium

- Retention of activity (as rate of lactic acid production) is relevant to cryoprotection studies

- Acid whey from cottage cheese production is a good medium for preserving frozen cultures

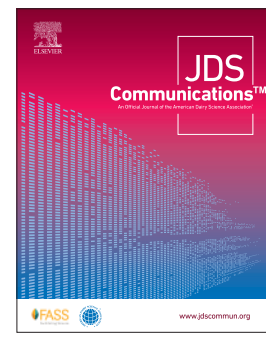




\title{
Effect of milk phospholipids on the growth and cryotolerance of lactic acid bacteria cultured and stored in acid whey-based media
}

\author{
Lin Zhang, ๑ Israel García-Cano, ๑ and Rafael Jiménez-Flores*๑
}

\begin{abstract}
Increasing interest in foods beyond their nutritional value has promoted the development of various novel functional foods that could convey multiple health benefits to consumers. The application of lactic acid bacteria (LAB) and milk phospholipids (MPL) in combination has shown some amplifying effects on the health benefits provided by both ingredients. Freezing is commonly used in $\mathrm{LAB}$ preservation and storage. However, the freezing/thawing process damages cell membranes and leads to a loss in viability and functionality of LAB. This study aimed to investigate the influence of MPL on growth and cryotolerance of LAB using acid whey-based medium (AWM) supplemented with $0.5 \%$ MPL. Fourteen LAB strains were initially screened from 124 LAB using acid whey-based medium (AW). We then evaluated the cell viability and acidification ability, using the plate counting method and skim milk fermentation test, respectively, of the 14 strains cultured in AW and AWM before and after 3 cycles of freezing/thawing. The presence of $0.5 \%$ MPL in AWM significantly promoted the growth of LAB. Supplementing the culture and storage medium with $0.5 \%$ MPL significantly enhanced the resistance of selected LAB to freeze-thaw cycles in terms of cell viability and acidification ability. These results suggest that supplementing with $0.5 \%$ MPL might promote the growth of LAB and enhance the cryotolerance of LAB cultures in fermented dairy products. This finding leads to a better understanding of the synergistic effects contributed by the LAB-MPL combination and promotes the development of new LAB-MPL functional products.
\end{abstract}

$M$ ilk phospholipids (MPL) are valuable dairy components of the milk fat globule membrane and have been associated with various health benefits, including promoting neurological development, protecting against inflammation, and inhibiting cancer cell proliferation (Snow et al., 2011; Castro-Gómez et al., 2016; Bhinder et al., 2017). Recently, MPL became commercially available and have been used as functional ingredients (Arranz and Corredig, 2017).

Lactic acid bacteria (LAB) have a long history of being used as starter cultures and probiotics. The most used commercial media for LAB culture are M17 and de Man, Rogosa, and Sharpe (MRS) broth. However, these media are expensive and are used exclusively for laboratory research. Acid whey has the potential to be used as an inexpensive alternative medium for culture of microorganisms. Acid whey is a by-product of the manufacture of fresh, acid-coagulated cheese (e.g., cottage cheese, cream cheeses) or strained yogurt (e.g., Greek-style yogurt) (Manley, 2000; Alsaed et al., 2013), which would cause serious environmental problems if not used or disposed of properly (Dudkiewicz et al., 2016). Acid whey is a valuable dairy stream with functional proteins and peptides, lactose, vitamin, and minerals, and it has great potential to be used as the main component in microorganism culture media (Bylund, 2015).

Because of the health benefits and compatibilities of MPL and LAB with dairy products, using LAB and MPL in combination has gained the attention of researchers and shown some synergistic effects. The LAB-MPL combination can amplify the health benefits compared with using the ingredients separately. Morifuji et al.
(2017) found that co-ingestion of LAB fermented milk and dietary sphingomyelin, a major group of MPL, increased the absorption of sphingomyelin in rats 2-fold, a significant increase compared with consuming sphingomyelin alone. Rocha-Mendoza et al. (2020) found that LAB previously cultured in MPL-supplemented medium exhibited greater adherence to Caco-2 cells. Moreover, the addition of 0.5 and $1 \%$ MPL into minimal medium promoted the growth of LAB, suggesting those LAB might utilize MPL as a source of energy or growth factors (Rocha-Mendoza et al., 2020). More investigations on the synergistic effects of the LAB-MPL combination are needed for the formulation of relevant functional products.

The optimal performance of LAB as both starter cultures and probiotics depends on the stabilization of their cell viability and metabolic activity. Frozen concentrates of LAB are extensively used in the manufacture of food, pharmaceutical, and dietary supplements. However, the freezing and thawing processes usually involved in preparation of frozen concentrates causes damage to cell membranes and leads to a loss in viability and functionality of $\mathrm{LAB}$. The cryotolerance of LAB refers to the resistance of bacteria to the freeze-thawing process. Higher cryotolerance would drive better retention in cell viability and acidification ability of bacteria after freezing (Wang et al., 2005; Meneghel et al., 2017). Little is known about the effects of MPL on the growth and cryotolerance of LAB. Exogenous fatty acid (FA) sources have been shown to promote the growth of LAB and enhance their cryotolerance (Williams et al., 1947; Partanen et al., 2001; Tan et al., 2012). Because of the FA groups present in MPL and their amphiphilic nature, 
we hypothesized that addition of MPL into LAB-fermented dairy products would promote the growth and enhance cryotolerance of LAB. In the present study, we investigated the effects of MPL on the growth and cryotolerance of LAB cultured and stored in acid whey-based media as measured by cell viability and acidification ability after 3 cycles of freeze-thawing treatment. This study may extend the use of the LAB-MPL combination because MPL is a valuable dairy ingredient that may protect $\mathrm{LAB}$ in both starter cultures and probiotics.

Fresh acid whey obtained after cottage cheese making from the Superior Dairy Company (Canton, $\mathrm{OH}$ ) was centrifuged at 10,000 $\times g$ for $20 \mathrm{~min}$ at $4^{\circ} \mathrm{C}$ (Sorvall Legend XT/XF Centrifuge; Thermo Scientific, Waltham, MA). After centrifugation, the supernatant was poured out and filtered using Whatman filter paper \#1. The permeate was deproteinized by autoclaving at $121^{\circ} \mathrm{C}$ for $15 \mathrm{~min}$, centrifuged, and filtered using the conditions as previously mentioned. Deproteinized whey was sterilized by autoclave at $121^{\circ} \mathrm{C}$ for 15 min to obtain basal acid whey. The prepared basal acid whey contained (wt/vol) 4.97 to $5.29 \%$ lactose, 0.55 to $0.69 \%$ protein, 0.06 to $0.08 \%$ fat, 0.48 to $0.52 \%$ ash, including 0.1 to $0.2 \%$ calcium. The prepared basal acid whey (AW) was supplemented with $0.5 \%(\mathrm{wt} / \mathrm{vol})$ autoclaved yeast extract (Sigma-Aldrich, St. Louis, $\mathrm{MO}$ ) and $0.5 \%(\mathrm{wt} / \mathrm{vol}) \mathrm{MPL}$ (Fonterra, Auckland, New Zealand) to make the treatment medium, AWM. The MPL ingredient has a purity of $60 \%$, so AWM contained $0.3 \mathrm{~g}$ of pure MPL per 100 $\mathrm{mL}$ of liquid medium. The MPL in AWM was replaced with the same amount of autoclaved reverse osmosis water (ThermoFisher Scientific, Waltham, MA) in the control medium (AW). The $\mathrm{pH}$ of the prepared AW and AWM was 4.5 to 4.6.

Normalized bacterial cultures of $124 \mathrm{LAB}$ strains from the OSUPECh culture collection (The Ohio State University, Columbus; García-Cano et al., 2019) were inoculated into AW by adjusting the initial absorbance (optical density) at $600 \mathrm{~nm}\left(\mathbf{O D}_{600}\right)$ to be 0.08 to 0.1 . The bacteria cultures were added into a sterile $96-$ well plate and incubated inside the AccuSkan GO UV/Vis Microplate Spectrophotometer (Fisher Scientific, Pittsburgh, PA) at $37^{\circ} \mathrm{C}$ for $18 \mathrm{~h}$ with a 1 -min pulsed medium shaking every $15 \mathrm{~min}$. The maximum $\mathrm{OD}_{600}$ of each strain was recorded (assayed in duplicate). Fourteen representative LAB strains with a maximum $\mathrm{OD}_{600}>0.4$ were selected for further experiments in this study: Pediococcus acidilactici OSU-PECh-1A; Lactobacillus amylolyticus OSUPECh-23A; Limosilactobacillus reuteri OSU-PECh-33A, OSUPECh-33B, OSU-PECh-35A, OSU-PECh-37A, OSU-PECh-48, OSU-PECh-50, OSU-PECh-81A, and OSU-PECh-92; Streptococcus thermophilus OSU-PECh-53; Lacticaseibacillus paracasei OSU-PECh-56; Lacticaseibacillus rhamnosus OSU-PECh-57A; and Lactobacillus acidophilus OSU-PECh-89.

Normalized bacteria cultures of those 14 selected LAB strains were inoculated into $5 \mathrm{~mL}$ of AW and AWM, respectively, with an initial $\mathrm{OD}_{600}$ of 0.08 to 0.1 . The bacteria cultures were incubated at $37^{\circ} \mathrm{C}$ for $24 \mathrm{~h}$. Then, bacteria were transferred one more time and inoculated $0.1 \%$ (vol/ $/ \mathrm{vol})$ into fresh AW or AWM and incubated at $37^{\circ} \mathrm{C}$ for $48 \mathrm{~h}$. After incubation, viable counts were done using MRS (Difco/Becton Dickinson Co., Sparks, MD) agar plates. The plates were incubated at $37^{\circ} \mathrm{C}$ for $36 \mathrm{~h}$ before counting. All bacteria were assayed in duplicate, and all plates were inoculated in triplicate.
The freeze-thawing treatment was modified from Meneghel et al. (2017). One milliliter of bacterial culture obtained after culturing in AW or AWM was transferred into a 2-mL sterile cryogenic tube (Fisher Scientific). The tube was immediately frozen at $-80^{\circ} \mathrm{C}$ for $23 \mathrm{~h}$ and then thawed at room temperature for $1 \mathrm{~h}$ before conducting the next freeze-thawing cycle and analysis. The freeze-thawing process was repeated 3 times before performing the following experiments.

As described by Meneghel et al. (2017), LAB counts were calculated before and after freeze-thawing cycles treatment. Counts (in $\mathrm{cfu} / \mathrm{mL}$ ) were conducted using the surface plating method. Samples were serially diluted in saline solution $(0.85 \% \mathrm{NaCl}, \mathrm{pH}$ $=7.0$ ) and appropriate dilutions, determined by preliminary experiments, were spread on MRS agar plates. All bacteria were assayed in duplicate; 2 dilutions and a triplicate for each dilution were tested for each sample. The plates were incubated at $37^{\circ} \mathrm{C}$ for $48 \mathrm{~h}$ before counting. Only plates with counts in the range of 30 to 300 cfu counted toward the final calculation. The retention in viability (\%) was calculated as follows:

$$
\text { Viability }=\frac{\log [\mathrm{cfu} / \mathrm{mL}]_{\text {after treatment }}}{\log [\mathrm{cfu} / \mathrm{mL}]_{\text {before treatment }}} \times 100 \%
$$

where $\log [\mathrm{cfu} / \mathrm{mL}]_{\text {before treatment }}$ refers to bacterial cell concentration obtained before the freeze/thaw cycle treatment, and $\log [\mathrm{cfu} / \mathrm{mL}]_{\mathrm{after}}$ treatment refers to cell concentration determined after the treatment.

The rate of $\mathrm{pH}$ decrease in skim milk (Difco/Becton Dickinson Co.) after addition of bacterial cultures was measured to evaluate the retention of acidification activity, according to Meneghel et al. (2017) with modifications. Twenty milliliters of skim milk (Difco/ Becton Dickinson Co.) was reconstituted from dry powder at 100 $\mathrm{g} / \mathrm{L}$, sterilized by autoclaving, and warmed to $37^{\circ} \mathrm{C}$ in an incubator. The bacteria after 3 cycles of freeze-thaw were assayed by using $4 \mathrm{~mL}$ of the corresponding bacterial culture as inoculum in $20 \mathrm{~mL}$ of sterile skim milk medium, and the $\mathrm{pH}$ was measured directly after $4 \mathrm{~h}$ of fermentation at $37^{\circ} \mathrm{C}$. Acidification activity (\%) was calculated as follows:

Acidification activity $=\frac{[\mathrm{pH} \text { decrease }]_{\text {after treatment }}}{[\mathrm{pH} \text { decrease }]_{\text {before treatment }}} \times 100 \%$,

where $[\mathrm{pH} \text { decrease }]_{\text {before treatment }}$ refers to the decrease in $\mathrm{pH}$ of the skim milk inoculated with the bacterial cultures before the freeze/thaw cycle treatment, and $[\mathrm{pH} \text { decrease }]_{\text {after treatment }}$ refers to the decrease in $\mathrm{pH}$ of the skim milk inoculated with bacterial cultures after treatment.

To compare bacterial growth in AW and AWM and viability and acidification ability after freeze-thawing treatment, we conducted a linear mixed-effect model with treatment as a fixed effect and bacteria as a random effect, with an $F$-test for each experiment (R soft- 
ware version 3.6.1 by lme4 package, https://www.r-project.org/). The criterion for significance of all tests was set at $P<0.05$.

The growth of LAB cultured using AW varied widely, as shown in Figure 1. A similar acid whey-based medium prepared in another study was found to create favorable conditions for the growth of some strains but not all because of its incomplete nutrient profile and low $\mathrm{pH}$ (4.5-4.6 in this study; Mondragón-Parada et al., 2006; Dudkiewicz et al., 2016). Usually, dominant dairy products that deliver LAB, such as yogurt and other fermented milk, have a relatively low pH (Ranadheera et al., 2010). Our prepared AW was similar to conditions that LAB would encounter in fermented dairy products and hence was appropriate for screening of LAB in our study. Fourteen $\mathrm{LAB}$ strains with a final $\mathrm{OD}_{600}>0.4$ were selected for later experiments. Because selected LAB could grow in prepared $\mathrm{AW}$ (with a final $\mathrm{OD}_{600}>0.4$ ), we could observe the effect of supplemented MPL on those LAB. In contrast, if LAB with final $\mathrm{OD}_{600}<0.4$ were selected, they would not grow well in prepared AW, and we could not observe the effects of MPL on those bacteria. These 14 strains were chosen because they were representative of growth in AW medium.

Statistical analysis of the bacterial count data showed that AWM resulted in a higher bacterial concentration than culture in AW
$(P$-value $=0.002)$, indicating that the presence of $0.5 \% \mathrm{MPL}$ in AWM significantly promoted growth of LAB. Previous studies have shown that a low concentration of medium- or long-chain UFA promoted growth of LAB, whereas a high concentration of long-chain SFA had inhibitory effects (Williams et al., 1947; Partanen et al., 2001). The AW medium had a limited amount of fat $(0.06-0.08 \%)$. Milk phospholipids are defined as "lipids containing phosphorus," with an amphiphilic structure consisting of a hydrophilic phosphorus head and a hydrophobic tail containing FA, mainly UFA (Contarini and Povolo, 2013; Ortega-Anaya and Jiménez-Flores, 2019). Hence, we might speculate that there are similarities between the effects of fats and FA on LAB and those caused by MPL, so it is reasonable that the supplementation of AW with $0.5 \% \mathrm{MPL}$ promoted growth. Another study found that the addition of $1 \%$ milk fat into Cheddar cheese resulted in a higher final cell density of Lacticaseibacillus casei (Tan et al., 2012).

After 3 freeze-thaw cycles, the viability of most tested LAB (92\%) previously cultured in both AW and AWM was $>70 \%$ (Figure 2), indicating good retention of viability of those tested $\mathrm{LAB}$ isolated from fermented dairy products in general. Statistical analysis of viability data demonstrated that bacteria cultured and stored in AWM had significantly higher viability than the same

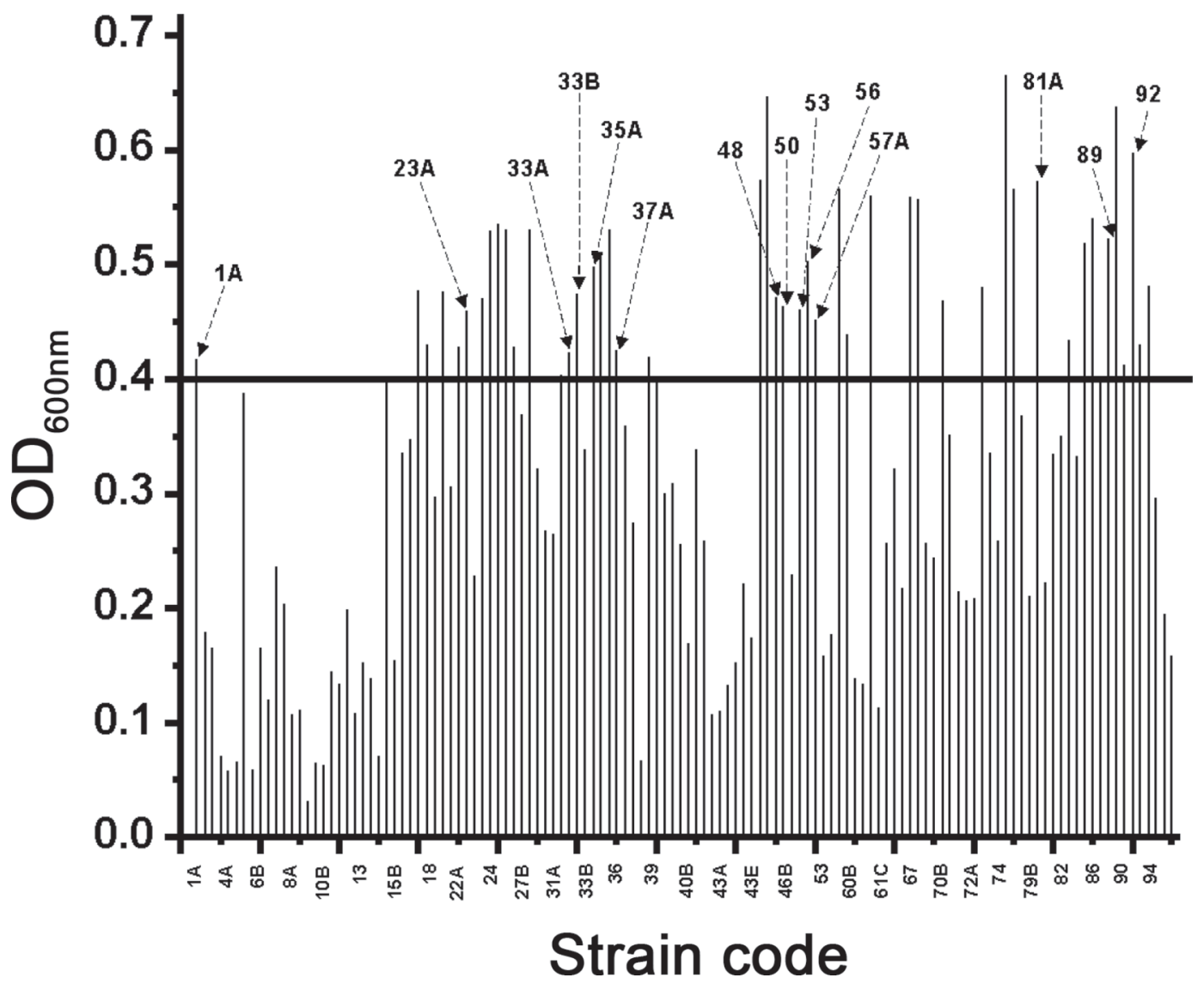

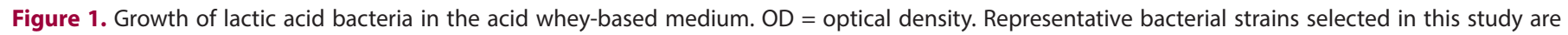

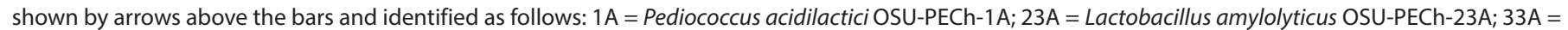

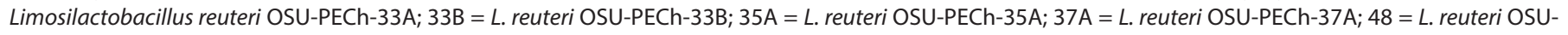

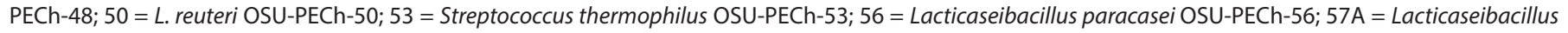
rhamnosus OSU-PECh-57A; 81A = L. reuteri OSU-PECh-81A; 89 = Lactobacillus acidophilus OSU-PECh-89; 92 = L. reuteri OSU-PECh-92. 


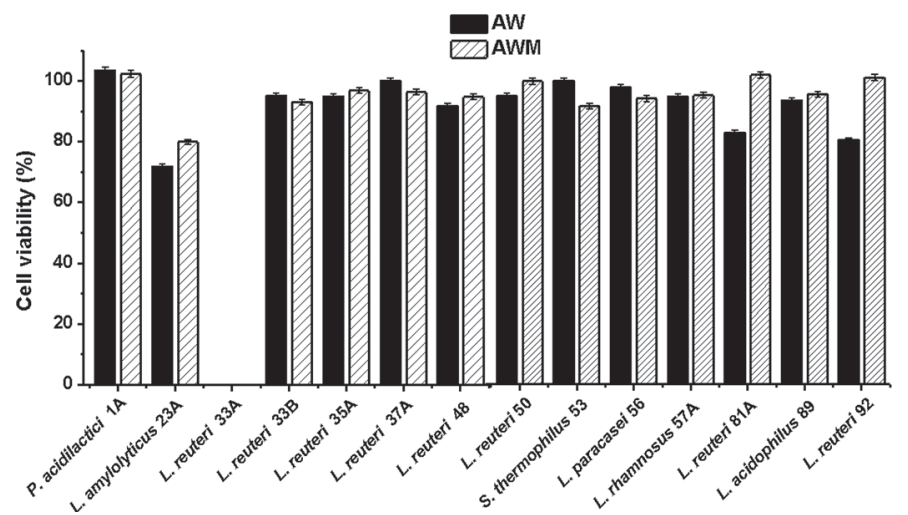

Figure 2. Comparison of cell viability of lactic acid bacteria (LAB) cultured and stored in acid whey-based medium (AW) and acid whey-based medium supplemented with $0.5 \%$ milk phospholipids (AWM) after freeze-thawing treatment. Strains: Pediococcus acidilactici OSU-PECh-1A, Lactobacillus amyIolyticus OSU-PECh-23A, Limosilactobacillus reuteri OSU-PECh-33A, L. reuter OSU-PECh-33B, L. reuteri OSU-PECh-35A, L. reuteri OSU-PECh-37A, L. reuteri OSU-PECh-48, L. reuteri OSU-PECh-50, Streptococcus thermophilus OSUPECh-53, Lacticaseibacillus paracasei OSU-PECh-56, Lacticaseibacillus rhamnosus OSU-PECh-57A, L. reuteri OSU-PECh-81A, Lactobacillus acidophilus OSU-PECh-89, and L. reuteri OSU-PECh-92. Error bars are standard deviations.

strain cultured and stored in $\mathrm{AW}(P=0.0123)$. Viability is a critical factor when incorporating probiotic LAB into dairy products. An adequate amount of viable probiotic cultures should be present in probiotic products throughout their shelf life to be able to confer health benefits to the host. It is generally accepted that for the probiotic effect to be transferred to the host, products should have a minimum concentration of $10^{6} \mathrm{cfu} / \mathrm{mL}$ or $10^{6} \mathrm{cfu} / \mathrm{g}$, and that a total of $10^{8}$ to $10^{9}$ probiotic microorganisms should be consumed daily (Kechagia et al., 2013). However, retention in metabolic activities cannot be reflected by cell viability: a bacterium can remain viable but lose its functional activity.

The retention of acidification ability, one important metabolic activity of $\mathrm{LAB}$, showed a wide range from 0 to $100 \%$ among tested strains (Figure 3). Statistical analysis of the acidification ability data demonstrated that bacteria cultured and stored in AWM retained acidification ability significantly better than the same strain cultured and stored in AW $(P<0.0001)$. The various degrees of damage to LAB caused by freeze-thaw cycles have been reported previously (Meneghel et al., 2017).

The effects of freeze-thaw cycles on LAB include inhibiting the growth of LAB, reducing or interrupting their metabolic activity, and even microorganism death, which affects the viability and functional properties of incorporated probiotic LAB (Alamprese et al., 2002). Modification of culture conditions; for example, temperature (Murga et al., 2000), $\mathrm{pH}$ (Wang et al., 2005), nutrients (Panoff et al., 2000), and the addition of cryoprotective agents to storage media or food products (e.g., glycerol, Fonseca et al., 2006; inulin, Rezaei et al., 2014), has been shown to improve the cryotolerance of LAB. Studies have also suggested that most LAB could incorporate exogenous FA such as oleic acid (Partanen et al., 2001; Tan et al., 2012) and PUFA (Kankaanpää et al., 2004) into their membranes, which could increase the fluidity of the membrane and

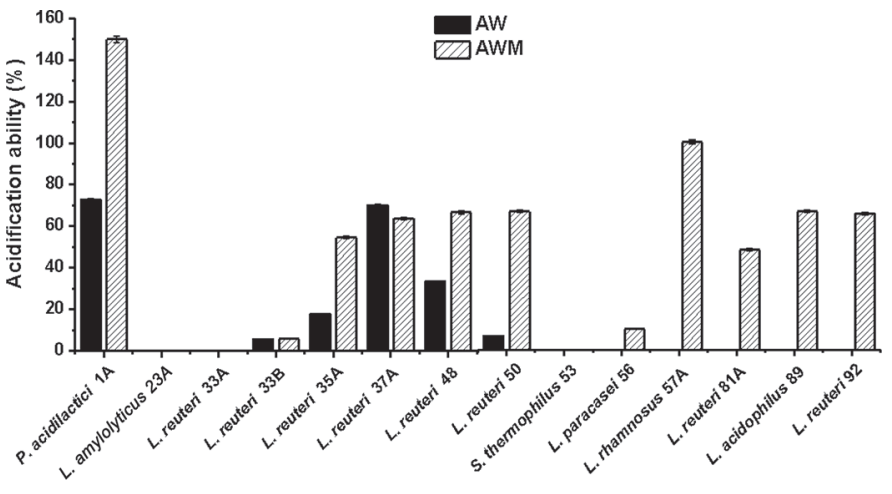

Figure 3. Comparison of acidification activity of lactic acid bacteria (LAB) cultured and stored in acid whey-based medium (AW) and acid whey-based medium supplemented with $0.5 \%$ milk phospholipids (AWM) after freeze-thawing treatment. Strains: Pediococcus acidilactici OSU-PECh-1A, Lactobacillus amylolyticus OSU-PECh-23A, Limosilactobacillus reuteri OSU-PECh-33A, L. reuteri OSU-PECh-33B, L. reuteri OSU-PECh-35A, L. reuteri OSU-PECh-37A, $L$. reuteri OSU-PECh-48, L. reuteri OSU-PECh-50, Streptococcus thermophilus OSU-PECh-53, Lacticaseibacillus paracasei OSU-PECh-56, Lacticaseibacillus rhamnosus OSU-PECh-57A, L. reuteri OSU-PECh-81A, Lactobacillus acidophilus OSU-PECh-89, and L. reuteri OSU-PECh-92. Error bars are standard deviations.

thus protect the LAB against damage caused by freeze-thawing and extreme temperature (Liu and Hofmann, 1962; Smittle et al., 1974; Partanen et al., 2001). Membrane fluidity has been correlated with storage temperature and a combination of different FA structures, including different saturation levels and chain lengths (Meneghel et al., 2017). Higher membrane fluidity provides bacteria with greater resistance to freezing and thawing stress (Kwon et al., 2018). Tan et al. (2012) found that the addition of $1 \%$ milk fat into Cheddar cheese would change the cytoplasmic membrane FA composition of Lacticaseibacillus casei. Because MPL are polar fractions of milk fat, LAB might be able to incorporate certain FA from the supplemented MPL, resulting in a change in cell membrane FA composition, and thus acquire higher cryotolerance. The cell membrane FA composition of MPL-treated LAB would be an interesting topic for future research. We think that this effect due to the interaction with MPL is strain-specific in the case of LAB, and likely also has some specificity in other bacteria.

In this study, AW medium is a way of utilizing acid whey and could support the growth of some $\mathrm{LAB}$ with a final $\mathrm{OD}_{600}>0.4$, which is high enough to observe the effect of MPL on LAB. Moreover, AW medium is similar to commercial fermented dairy products, so it is more appropriate for assessing effects of MPL than MRS medium. The results of this study support the conclusion that addition of $0.5 \%$ MPL to AW promotes the growth and enhances the cryotolerance of LAB significantly, specifically in terms of cell viability and acidification ability. This study may lead to a better understanding of the synergistic effects of using MPL and $\mathrm{LAB}$ in combination and may contribute to the production of novel functional products.

\section{References}

Alamprese, C., R. Foschino, M. Rossi, C. Pompei, and L. Savani. 2002. Survival of Lactobacillus johnsonii Lal and influence of its addition in 
retail-manufactured ice cream produced with different sugar and fat concentrations. Int. Dairy J. 12:201-208. https://doi.org/10.1016/S0958 -6946(01)00159-5.

Alsaed, A. K., R. Ahmad, H. Aldoomy, S. Abd El-Qad, D. Saleh, H. Sakejha, and L. Mustafa. 2013. Characterization, concentration and utilization of sweet and acid whey. Pak. J. Nutr. 12:172-177. https://doi.org/10.3923/ pjn.2013.172.177.

Arranz, E., and M. Corredig. 2017. Invited review: Milk phospholipid vesicles, their colloidal properties, and potential as delivery vehicles for bioactive molecules. J. Dairy Sci. 100:4213-4222. https://doi.org/10.3168/jds.2016 -12236 .

Bhinder, G., J. M. Allaire, C. Garcia, J. T. Lau, J. M. Chan, N. R. Ryz, E. S. Bosman, F. A. Graef, S. M. Crowley, L. S. Celiberto, J. C. Berkmann, R. A. Dyer, K. Jacobson, M. G. Surette, S. M. Innis, and B. A. Vallance. 2017. Milk fat globule membrane supplementation in formula modulates the neonatal gut microbiome and normalizes intestinal development. Sci. Rep. 7:45274. https://doi.org/10.1038/srep45274.

Bylund, G. 2015. Dairy Processing Handbook. Tetra Pak Processing Systems AB. Accessed February 6, 2020. https://dairyprocessinghandbook.tetrapak .com/.

Castro-Gómez, P., L. M. Rodríguez-Alcalá, K. M. Monteiro, A. L. T. G. Ruiz, J. E. Carvalho, and J. Fontecha. 2016. Antiproliferative activity of buttermilk lipid fractions isolated using food grade and non-food grade solvents on human cancer cell lines. Food Chem. 212:695-702. https://doi.org/10 .1016/j.foodchem.2016.06.030.

Contarini, G., and M. Povolo. 2013. Phospholipids in milk fat: Composition, biological and technological significance, and analytical strategies. Int. J. Mol. Sci. 14:2808-2831. https://doi.org/10.3390/ijms14022808.

Dudkiewicz, M., J. Berlowska, and D. Kregiel. 2016. Acid whey as a medium for cultivation of conventional and non-conventional yeasts. Biotechnol. Food Sci. 80:75-82.

Fonseca, F., M. Marin, and G. J. Morris. 2006. Stabilization of frozen Lactobacillus delbrueckii ssp. bulgaricus in glycerol suspensions: Freezing kinetics and storage temperature effects. Appl. Environ. Microbiol. 72:6474-6482. https://doi.org/10.1128/AEM.00998-06.

García-Cano, I., D. Rocha-Mendoza, J. Ortega-Anaya, K. Wang, E. Kosmerl, and R. Jiménez-Flores. 2019. Lactic acid bacteria isolated from dairy products as potential producers of lipolytic, proteolytic and antibacterial proteins. Appl. Microbiol. Biotechnol. 103:5243-5257. https://doi.org/10 .1007/s00253-019-09844-6.

Kankaanpää, P., B. Yang, H. Kallio, E. Isolauri, and S. Salminen. 2004. Effects of polyunsaturated fatty acids in growth medium on lipid composition and on physicochemical surface properties of lactobacilli. Appl. Environ. Microbiol. 70:129-136. https://doi.org/10.1128/AEM.70.1.129-136.2004.

Kechagia, M., D. Basoulis, S. Konstantopoulou, D. Dimitriadi, K. Gyftopoulou, N. Skarmoutsou, and E. M. Fakiri. 2013. Health benefits of probiotics: A review. ISRN Nutr. 2013:481651. https://doi.org/10.5402/2013/481651.

Kwon, Y. W., J.-H. Bae, S.-A. Kim, and N. S. Han. 2018. Development of freeze-thaw tolerant Lactobacillus rhamnosus GG by adaptive laboratory evolution. Front. Microbiol. 9:2781. https://doi.org/10.3389/fmicb.2018 .02781 .

Liu, T. Y., and K. Hofmann. 1962. Cyclopropane ring biosynthesis. Biochemistry 1:189-191. https://doi.org/10.1021/bi00907a028.

Manley, D. 2000. Chapter 13: Milk products and egg. Pages 161-168 in Technology of Biscuits, Crackers and Cookies. 3rd ed. Woodhead Publishing Series in Food Science, Technology and Nutrition Book. Elsevier, Amsterdam, the Netherlands.

Meneghel, J., S. Passot, S. Dupont, and F. Fonseca. 2017. Biophysical characterization of the Lactobacillus delbrueckii ssp. bulgaricus membrane during cold and osmotic stress and its relevance for cryopreservation. Appl. Microbiol. Biotechnol. 101:1427-1441. https://doi.org/10.1007/s00253 -016-7935-4.

Mondragón-Parada, M. E., M. Nájera-Martínez, C. Juárez-Ramírez, J. Galíndez-Mayer, N. Ruiz-Ordaz, and E. Cristiani-Urbina. 2006. Lactic acid bacteria production from whey. Appl. Biochem. Biotechnol. 134:223-232. https://doi.org/10.1385/ABAB:134:3:223.

Morifuji, M., M. Kitade, C. Oba, T. Fukasawa, K. Kawahata, T. Yamaji, Y. Manabe, and T. Sugawara. 2017. Milk fermented by lactic acid bacteria enhances the absorption of dietary sphingomyelin in rats. Lipids 52:423-431. https://doi.org/10.1007/s11745-017-4247-0.

Murga, M. L. F., G. M. Cabrera, G. F. de Valdez, A. Disalvo, and A. M. Seldes. 2000. Influence of growth temperature on cryotolerance and lipid composition of Lactobacillus acidophilus. J. Appl. Microbiol. 88:342-348. https:// doi.org/10.1046/j.1365-2672.2000.00967.x.

Ortega-Anaya, J., and R. Jiménez-Flores. 2019. Symposium review: The relevance of bovine milk phospholipids in human nutrition-Evidence of the effect on infant gut and brain development. J. Dairy Sci. 102:2738-2748. https://doi.org/10.3168/jds.2018-15342.

Panoff, J.-M., B. Thammavongs, and M. Guéguen. 2000. Cryoprotectants lead to phenotypic adaptation to freeze-thaw stress in Lactobacillus delbrueckii ssp. bulgaricus CIP 101027T. Cryobiology 40:264-269. https://doi.org/10 $.1006 /$ cryo.2000.2240.

Partanen, L., N. Marttinen, and T. Alatossava. 2001. Fats and fatty acids as growth factors for Lactobacillus delbrueckii. Syst. Appl. Microbiol. 24:500-506. https://doi.org/10.1078/0723-2020-00078.

Ranadheera, R. D. C. S., S. K. Baines, and M. C. Adams. 2010. Importance of food in probiotic efficacy. Food Res. Int. 43:1-7. https://doi.org/10.1016/j .foodres.2009.09.009.

Rezaei, R., M. Khomeiri, M. Aalami, and M. Kashaninejad. 2014. Effect of inulin on the physicochemical properties, flow behavior and probiotic survival of frozen yogurt. J. Food Sci. Technol. 51:2809-2814. https://doi .org/10.1007/s13197-012-0751-7.

Rocha-Mendoza, D., E. Kosmerl, G. Miyagusuku-Cruzado, M. M. Giusti, R. Jimenez-Flores, and I. García-Cano. 2020. Growth of lactic acid bacteria in milk phospholipids enhances their adhesion to Caco-2 cells. J. Dairy Sci. 103:7707-7715. https://doi.org/10.3168/jds.2020-18271.

Smittle, R. B., S. E. Gilliland, M. L. Speck, and W. M. Walter Jr.. 1974. Relationship of cellular fatty acid composition to survival of Lactobacillus bulgaricus in liquid nitrogen. Appl. Microbiol. 27:738-743. https://doi .org/10.1128/AEM.27.4.738-743.1974.

Snow, D. R., R. E. Ward, A. Olsen, R. Jimenez-Flores, and K. J. Hintze. 2011. Membrane-rich milk fat diet provides protection against gastrointestinal leakiness in mice treated with lipopolysaccharide. J. Dairy Sci. 94:22012212. https://doi.org/10.3168/jds.2010-3886.

Tan, W. S., M. F. Budinich, R. Ward, J. R. Broadbent, and J. L. Steele. 2012. Optimal growth of Lactobacillus casei in a Cheddar cheese ripening model system requires exogenous fatty acids. J. Dairy Sci. 95:1680-1689. https:/ /doi.org/10.3168/jds.2011-4847.

Wang, Y., G. Corrieu, and C. Béal. 2005. Fermentation pH and temperature influence the cryotolerance of Lactobacillus acidophilus RD758. J. Dairy Sci. 88:21-29. https://doi.org/10.3168/jds.S0022-0302(05)72658-8.

Williams, W. L., H. P. Broquist, and E. E. Snell. 1947. Oleic acid and related compounds as growth factors for lactic acid bacteria. J. Biol. Chem. 170:619-630.

\section{Notes}

Lin Zhang @ https://orcid.org/0000-0002-8956-2768

Israel García-Cano ำ https://orcid.org/0000-0003-2891-7286

Rafael Jiménez-Flores @ https://orcid.org/0000-0003-4905-5021

This work was funded by the Parker Endowment (Department of Food Science and Technology, The Ohio State University, Columbus).

The authors thank Molly Davis (Department of Food Science and Technology, The Ohio State University, Columbus) for her editing.

The authors have not stated any conflicts of interest. 